\title{
Hypercoagulability in COVID-19
}

\section{Pieter Wessels}

Ampath Laboratories, Pretoria, South Africa

Department of Medical Oncology, Steve Biko Academic Hospital,

Pretoria, South Africa

Department of Haematology, University of Pretoria, Pretoria,

South Africa

Address for correspondence:

Dr Pieter Wessels

Life Groenkloof Hospital

50 George Storrar Drive

Groenkloof

Pretoria

$0|8|$

South Africa

Email:

wesselspf@mweb.co.za

\section{INTRODUCTION}

On the last day of 2019, China alerted the WHO of several cases of unusual pneumonia in Wuhan by an unknown virus. More than 40 infections were noted in workers from the city's Huanan Seafood Wholesale Market, which was shut down on the first day of 2020. The first death (heart failure as cause) occurred officially on 9 January 2020. Within 2 weeks, similar cases were reported from Thailand and several other countries. A month after the first alert, on 30 January 2020, the WHO declared the coronavirus a global emergency. ${ }^{(1)}$ Since then, more than 6 million people have been known to be infected, with more than 360000 reported deaths (at time of writing, 28 May 2020), and in South Africa more than 26000 positive cases have been reported,(2) with the first death in South Africa possibly due to possible pulmonary embolism. The coronavirus disease of 2019 (COVID-19) is caused by the severe acute respiratory syndrome coronavirus 2 (SARSCoV-2). Although based on scarce direct evidence, but mostly observations/experiences of clinicians treating these patients (clinical trials are only being done at time of writing), the following observations have been made:

Initially patients may experience the clinical picture of a viremia with mild constitutional symptoms of fever, fatigue, headache, cough, myalgia, occasionally with gastro-intestinal symptoms

\section{ABSTRACT}

COVID-I9 is associated with a hypercoagulable state that may present as pulmonary thrombosis, pulmonary embolism, and venous and arterial thrombosis.

Suggested pathogenesis include direct infection of the endothelial cell with subsequent endothelial cell dysfunction, leading to increased procoagulant activity, decreased anticoagulant activity and decreased fibrinolysis. The severe immune inflammatory response in the lungs with cytokine release also plays a critical role (immunothrombosis). Hypoxia has a local and systemic effect on coagulation. Various markers of this state have been described, and especially the D-dimer level (and rapid changes in the D-dimer level) as a reliable prognostic marker. It is also used as indicator for initiation of anticoagulation by some experts. Due to the pleotrophic effects of heparin, it is the anticoagulant of choice for these patients (most often low molecular weight heparin, due to decreased risk of heparin induced thrombocytopenia, ease of use). No clinical trial data is available at the time of writing (28 May 2020), and suggested guidelines of experts in different countries are discussed. SAHeart 2020;1 7:266-274

such as diarrhoea, and some with neurological symptoms (most commonly anosmia). In this phase of the disease, laboratory abnormalities may already be present, with markers indicating increased risk of severe disease and potential for progression to mechanical ventilation or ICU admission. ${ }^{(3,4)}$

\section{These include:}

Lymphopenia (and neutrophil:lymphocyte ratio)

- Increased D-dimer values $(>0.5 \mathrm{mg} / \mathrm{L}$ or $>$ age-adjusted value)

- Mildly prolonged prothrombin time

- High fibrinogen degradation products (FDP) values

Increased LDH levels

A proportion of patients with clinical markers predictive of more severe disease such as older age, male gender, comorbidities such as hypertension and diabetes, cancer or high BMI, may develop pulmonary symptoms such as dyspnea and mild hypoxemia. In this phase, a rising C-reactive protein (CRP) is 
present, with low procalcitonin.(5) D-dimer levels are increased and high acute phase reactant levels such as ferritin and interleukin-6 (IL-6) are noted.

An even smaller proportion of patients may progress to the third hyperinflammation or cytokine storm, with very high IL-6 levels, very high ferritin levels and very high D-dimer (even $>3 \mathrm{mg} / \mathrm{L}$ or $6 \times$ upper limit of normal) levels. This leads to organ dysfunction including the acute respiratory distress syndrome, hypotension, cardiac failure, and renal dysfunction. In the terminal stages of the disease, criteria compatible with disseminated intravascular clotting (DIC) may be present - such as thrombocytopenia and low fibrinogen level. (6)

A hypercoagulable state is present during the course of the disease, and coagulopathy is associated with high mortality. ${ }^{(7)}$ Initially, in China, a few reports of venous thrombosis were described, ${ }^{(8)}$ but with the spread to Europe, various reports of venous $^{(9)}$ as well as arterial thrombosis ${ }^{(10)}$ have been reported. An incidence of $31 \%$ of VTE has been described in the sick ICU COVID-19 patients, despite standard thromboprophylaxis. ${ }^{(9)}$ Arterial thrombosis may be due to the systemic inflammatory response and vascular inflammation associated with vascular plaques. Formation of microvascular thrombi in the pulmonary capillaries has been suggested to be part of the pathogenesis of the disease. This pulmonary specific vasculopathy has been termed pulmonary intravascular coagulopathy (PIC).(II) Anticoagulant therapy is associated with decreased mortality in severe COVID cases. ${ }^{(12)}$ Recent publications describing autopsy findings have confirmed the presence of thrombosis in small and mid-sized pulmonary arteries in all the patients examined, with pulmonary infarction in more than $70 \%$ of patients. ${ }^{(13)}$ In another recent paper (prospective study on the first 12 deaths in a German academic centre), 58\% of patients at autopsy showed venous thrombo-embolism, in whom VTE that had not been suspected prior to death, with PE as the direct cause of death in $33 \%$ of patients. ${ }^{(14)}$

\section{SEVERAL QUESTIONS ARISE FROMTHESE OBSERVATIONS:}

What is the pathogenesis of this hypercoagulable state, and is this different from other inflammatory states? Four different processes (which are interactive and interdependent) play a role in thrombosis and haemostasis (with some of the major role players) (Figure I). These consist of:

\section{Procoagulant activity}

Coagulation factors (CF) circulate in an inactive form, except for a small percentage of active Factor VII (FVIla). Contact with FIII [tissue factor (TF)], which is present in tissue as well as monocytes, activates the clotting cascade leading to the formation of fibrin strands. Thrombin (Flla) plays the part of conductor of this system. FXlla, which is activated by surface contact, including platelets, activates the pro-inflammatory kallikrein-kininogen pathway.

Platelet surfaces have receptors for subendothelial structures, and when activated, release content such as von Willebrand factor (VWF), Factor V, Factor VIII, PAI-I, chemokines,

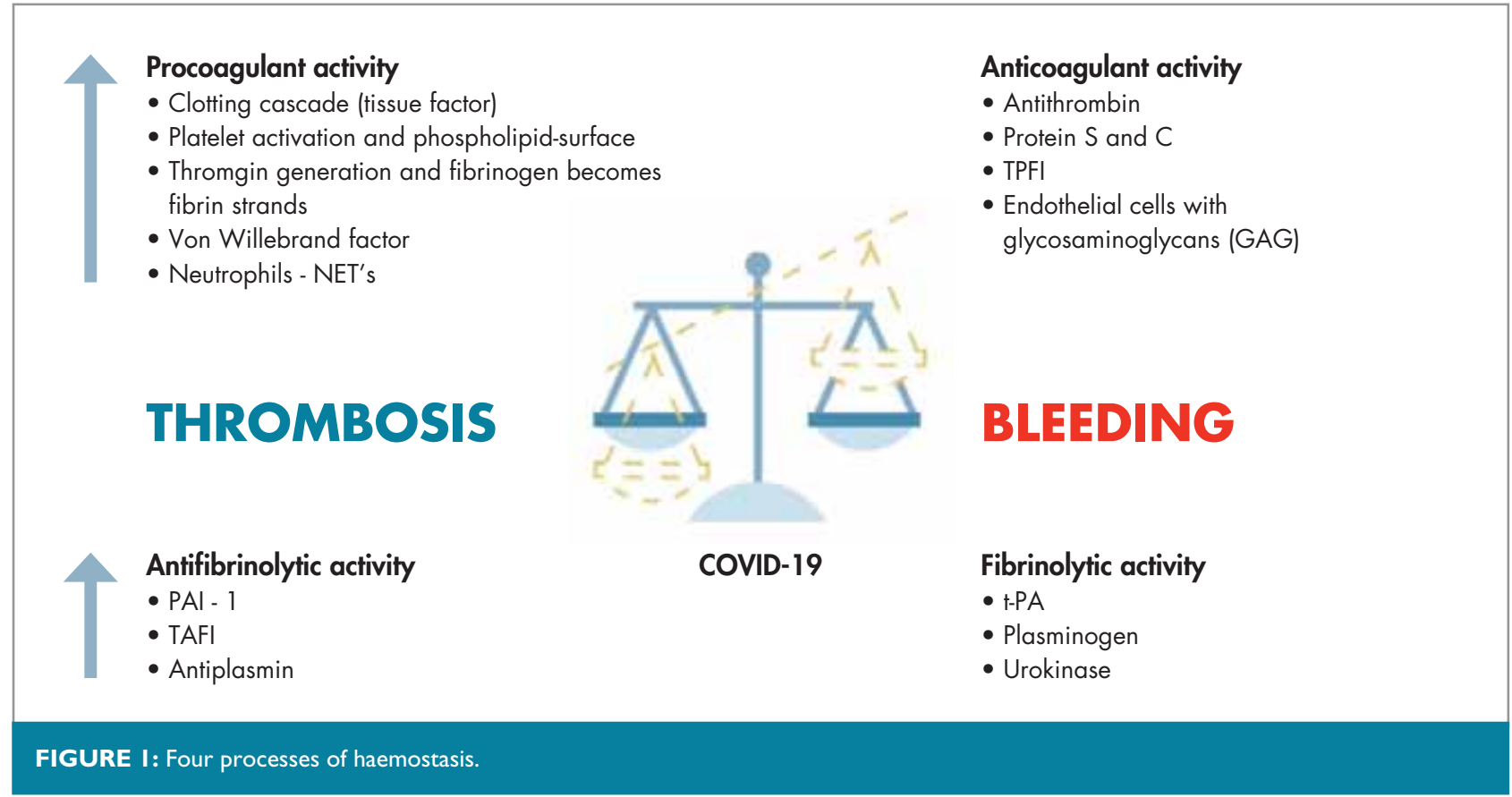


fibrinogen, calcium, ATP and ADP. Platelets thus have a microbicidal and chemotactic role, supply the phospholipid microparticles that are needed for the coagulation pathway, and supply clotting factors and activate other platelets.

Neutrophils are known to produce neutrophil extracellular traps (NETs), which are fibrous nets with antimicrobial properties capable of killing bacteria and fungi, and which are also released in viral infections.

Von Willebrand factor (VWF) is a multimer adhesive glycoprotein present beneath the endothelial cells (to which blood platelets can bind). Large multimers of VWF are released by endothelial cells under conditions of sheer stress conditions, but are quickly cleaved by a metalloproteinase (ADAMTS-13).

\section{Anticoagulant activity}

Endothelial cells (EC) (see Figure 2) form a smooth barrier between blood and subendothelial structures, control vasomotor tone by release of prostacyclin and nitrous oxide, and when activated release and express adhesion molecules on the inner surface that control cellular traffic (inflammatory cell and platelet adhesion and vascular permeability). ECs also play an important part in the fibrinolytic pathway by manufacturing tissue plasminogen activator (tPA) and plasminogen activator inhibitor (PAI-I). EC have a role in activating circulating anticoagulants such as protein $\mathrm{C}$, and even have a role in antifibrinolytic activity by activating thrombin activatable fibrinolysis inhibitor (TAFI).

Circulating anticoagulants include antithrombin, which inhibits thrombin, FIXa, FXa, FXla and FXIIla, protein S and protein C which inhibit FVa and FVIIla, and tissue factor pathway inhibitor which inhibits FXa.

\section{Fibrinolytic activity}

Tissue plasminogen activator (tPA) is a protease that is secreted by ECs (stimuli for this includes venous occlusion, thrombin (Flla) and strenuous exercise), which converts plasminogen to plasmin. Plasmin hydrolyses fibrinogen and fibrin, with resultant D-dimer peptides.

\section{Anti-fibrinolytic activity}

Plasminogen activator inhibitor (PAI-I) is secreted by ECs, and it inhibits tPA. Thrombin activatable fibrinolysis inhibitor (TAFI)

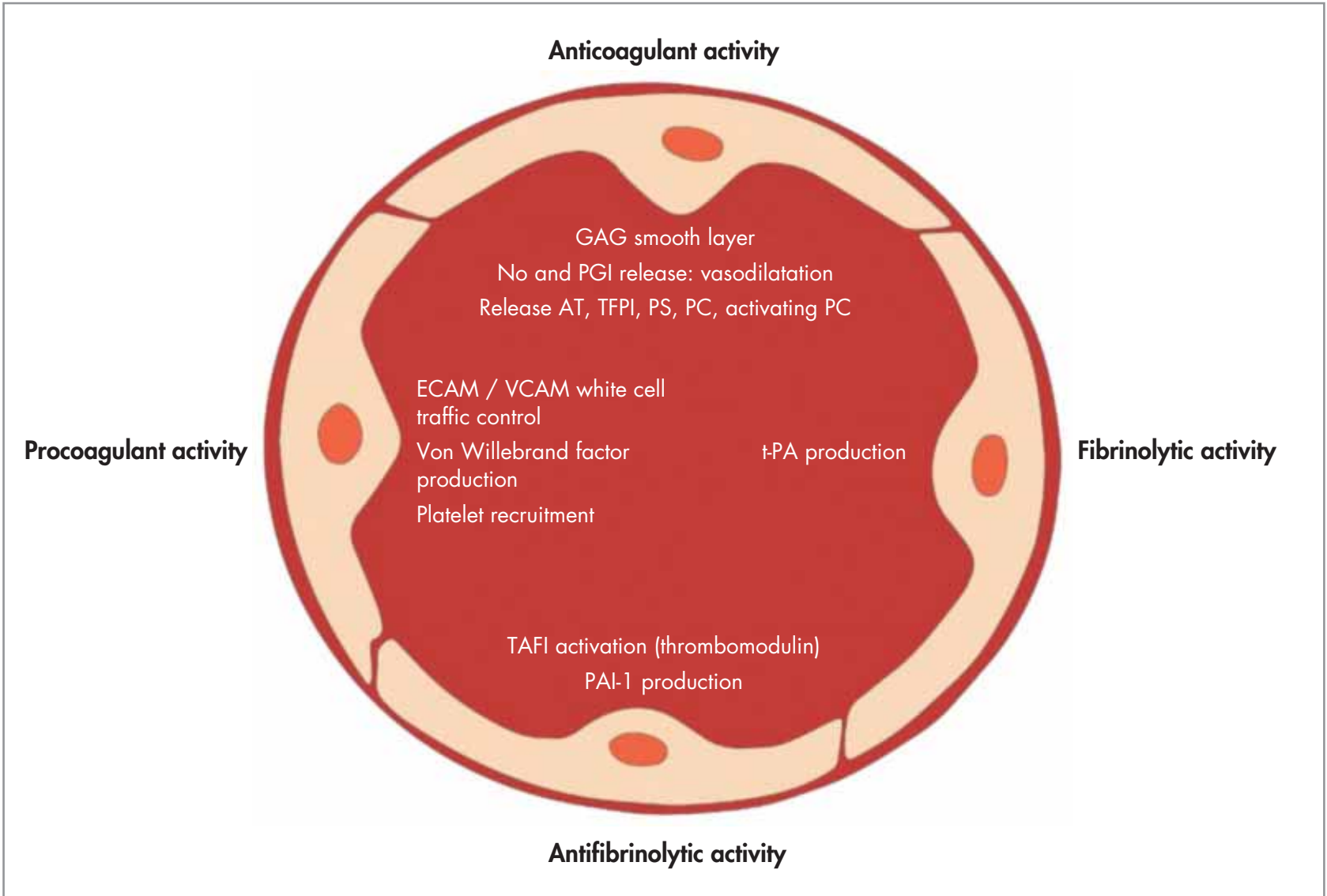

FIGURE 2: Endothelial cell role in haemostasis and thrombosis, depending on activation status. 
removes the lysine binding sites of fibrin for plasminogen and tPA, thus downregulating fibrinolytic activity.

Of note is the important role of the endothelial cell in all 4 processes (Figure 2).

\section{Two further concepts are also important:}

There is a strong link between inflammation and thrombosis: Cytokine orchestration of both thrombus generation [Interleukin 6 (IL-6), IFN-Y, IL- I7, IL-9 and TGF- $\beta$ ], as well as thrombus resolution (IL-10, TNF-a and IL-8) have been reviewed recently. ${ }^{(15)}$ This inflammatory response includes activation of neutrophils (and thus NET formation), monocytes (with TF expression), platelets (with aggregation) and endothelial cells (with release of large von Willebrand factor multimers which overwhelm the metalloprotease ADAMTS- 13). ${ }^{(16)}$

The effect of hypoxia (local and systemic) on coagulation: Hypoxia-inducible transcription factors (HIFs) control the vascular response, with increased expression of tissue factor expression and PAI-I, as well as decreased TFPI and protein S expression, thus tipping the scale towards a hypercoagulable state. Simultaneously, hypoxia also increases the inflammatory response and activates platelets. ${ }^{(17)}$

\section{Suggested pathogenesis of hypercoagulability in COVID-I9 (Figure 3):}

The SARS-CoV-2 virus infects the host by attaching the surface spike glycoproteins to the angiotensin-converting enzyme 2 receptor (ACE-2 receptor) on host cells. The ACE-2 receptors are present in many organs, such as lung alveolar cells, heart, intestine and kidney podocytes and tubule epithelial cells, but also on endothelial cells and pericytes in the vicinity of the endothelial cell and lymphocytes. The inhaled virus initially attaches to the alveolar cells in the lungs and leads to pneumonia with an intense inflammatory response, precipitating an exudate of fibrinogen into the alveoli, with fibrin and hyaline membrane formation (seen in SARS as well as COVID-19). The inflammation in the alveolar cells triggers endothelial dysfunction, which leads to vasoconstriction and a procoagulant state in the vessel, with impaired microcirculation and organ ischaemia, recently termed Corona-19-associated hemostasis abnormality (CAHA). ${ }^{(18)}$ Evidence has been found of direct viral infection of the endothelial cell, causing diffuse inflammation of the cells ("endothelialitis"). ${ }^{(19)}$ Due to the sepsis and the inflammatory response, monocytes are activated and their TF expression activate the coagulation cascade. Platelets are also activated with resultant release of content and microparticles. Neutrophils are attracted to the area with NET formation (NETosis). These NETs activate the coagulation cascade (electrostatic interactions between NET histones and platelet phospholipids activate the "alternate pathway" via the kallikrein-kinin system). ${ }^{(20)}$ Monocytes (macrophages) in the area are induced to form ILI $\beta$ (which induces IL-6 production), and when overwhelmed by the ensuing cytokine storm, this loop leads to an uncontrolled progressive inflammatory process. ${ }^{(20)}$ In addition, large VWF multimers are released from the endothelial cell. All these procoagulant factors leads to a local hypercoagulable state with clot formation. Whether a different mechanism is involved in COVID-19 infections relative to other causes of severe sepsis, is uncertain, but the coexistent hypoxia (see above), may play an important role in determining its severity. ${ }^{(16)}$ The ACE2 receptor is part of the renin-angiotensin-aldosterone system (RAAS). After infection with COVID-19, ACE2 is downregulated, with an increase in angiotensin II (Ang II) levels. This leads to an increase in PAI-I, with inhibition of fibrinolysis. Even though the kinin-bradikinin pathway is activated with an associated increase in tPA, the scale is tipped towards hypercoagulability with increased PAI-I and decreased fibrinolysis.(21) In one study, ${ }^{(7)} 71 \%$ of patients that died of COVID-19, fulfilled the criteria for disseminated intravascular coagulation (DIC). This occurred at a late stage of the disease with associated thrombocytopenia, hypofibrinogenemia, prolonged PT, increased D-dimer value, and high SOFA (sequential organ failure assessment) score. The important entity of sepsisassociated coagulopathy (SIC) has been defined by the DIC standardisation subcommittee in 2019,(22) and is characterised by suppression of fibrinolysis, induced by endothelial dysfunction (as described above). The importance of this distinction is that anticoagulant therapy may improve outcomes in this early group of patients that eventually develop overt DIC, as SIC procedes DIC (see Figure 3). The presence of antiphospholipid antibodies in patients with COVID-19 has also been demonstrated, and even could be associated with catastrophic antiphospholipid syndrome (APLS). ${ }^{(23)}$

In view of the increased D-dimer, fibrinogen, ferritin and anaemia (schistocytes are not really noted on blood smears though) in association with renal dysfunction, fever and clear endothelial cell dysfunction, the pathogenesis of COVID-19 injury has also been suggested to be due to a secondary thrombotic micro-angiopathy (TMA). ${ }^{(24)}$ COVID-19 causes alterations in complement regulatory proteins with activation of the complement system, and at the same time infects pericytes and endothelial cells, leading to endothelial cell dysfunction.

\section{Why is the hypercoagulable state so important in COVID-19?}

The hypercoagulability, especially when localised to the lungs as part of the innate defense mechanism directed against the virus, may be an important part to the pathogenesis of the clinical picture, as such early anticoagulation has the potential to mediate the inflammatory process. It is important to distinguish pulmonary thrombosis due to the localised hypercoagulability from pulmonary embolism that may be seen in other ICU patients. In fact, ultrasound of the legs is often normal, and 


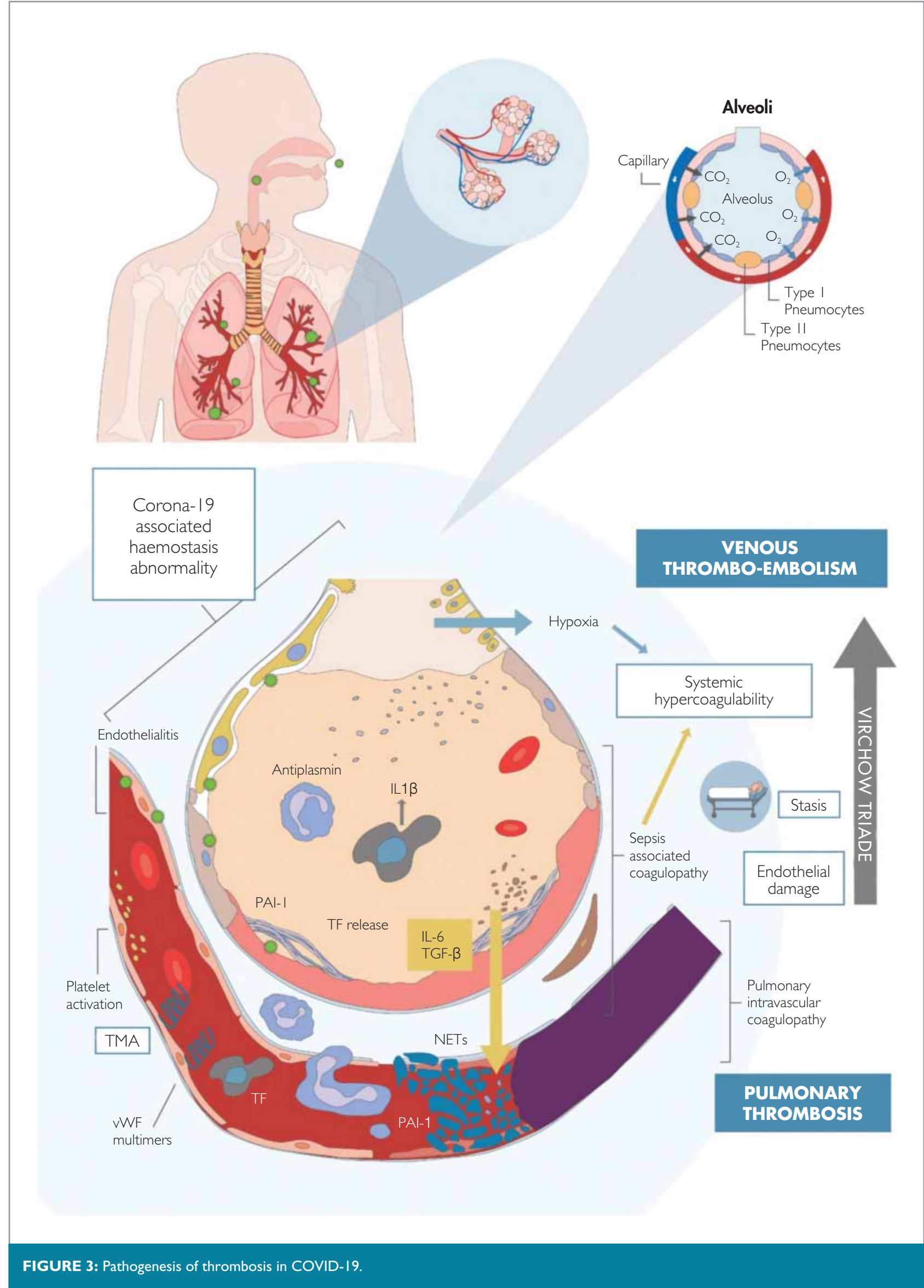


only by recognition that the hypoxia may not only be due to the pneumonia, can the diagnosis of pulmonary thrombosis be made.(14) Both intra-pulmonary thrombosis and classical pulmonary embolism may occur, as once immobilised and mechanically ventilated, all of Virchow's triad are present: stasis, endothelial damage and systemic hypercoagulability. Treatment may, however, differ between intra-pulmonary thrombosis and pulmonary embolism. With pulmonary embolism, anticoagulant therapy is indicated, but with the important role of platelets in pulmonary thrombosis, antiplatelet therapy may be of value, as well as nebulised antithrombotic therapies in pulmonary thrombosis. ${ }^{(18)}$

\section{How do we manage this hypercoagulable state?}

As no clinical trial data are currently available, there is uncertainty as to the timing and dosing of prophylactic anticoagulation. Some suggest using D-dimer values and weight-adjusted dosing, whereas others suggest different dosages at different stages of the disease, but also taking D-dimer values into consideration.
There are only interim guidelines at the time of writing, but it must be emphasised that prior to therapy, bleeding risk should first be evaluated.

\section{Timing and dose of anticoagulation (see Figure 4)}

Patients in the viremic phase, and in quarantine at home, should receive no anticoagulation but are advised to stay active as suggested by interim ISTH guidelines. ${ }^{(25)}$ If these patients have an underlying hypercoagulable state such as congenital thrombophilia, previous VTE, underlying malignancy, or any additional risk factor, standard prophylactic anticoagulation is recommended in countries such as United Kingdom, Spain and France. In France, Spain and Italy, it is recommended that obese patients with additional risk factors receive intermediate doses of VTE prophylaxis. D-dimer, CRP and ferritin values may help in the decision. A D-dimer value of more than $0.5 \mathrm{mg} / \mathrm{L}$ (or above age-related value cut-off) indicates an increased thrombotic risk, and an increased CRP and ferritin may indicate progression of the disease. Pregnant patients are already

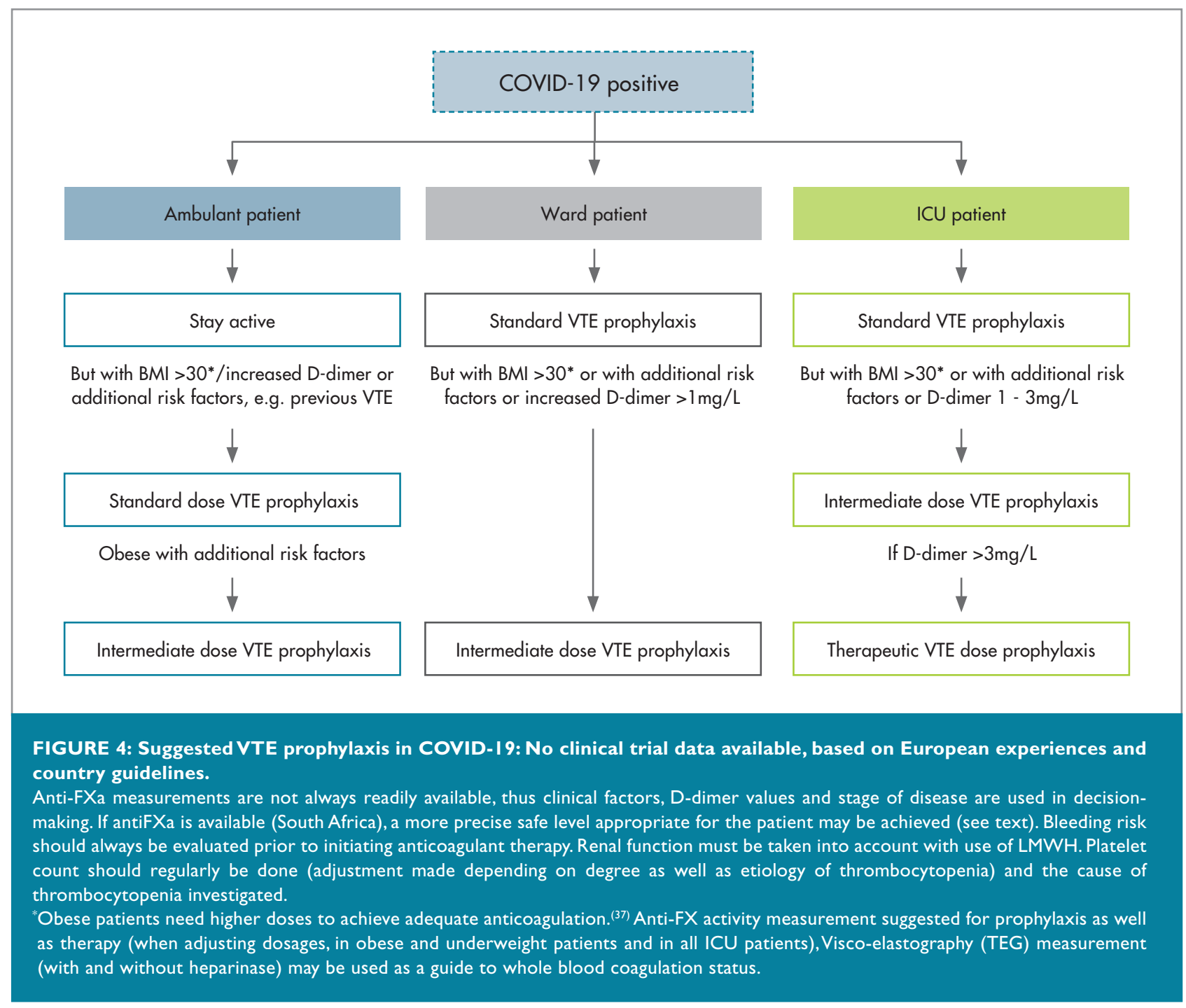


hypercoagulable and should also receive VTE prophylaxis (they should be put on the ISTH registry if possible: https://redcap. isth.org/surveys/?s=4JPX9W98RH).

Patients admitted to hospital should receive prophylactic thromboprophylaxis, irrespective of other additional risk factors present. If additional risk factors are known, such as obesity, previous VTE, and underlying malignancy, intermediate dose prophylactic anticoagulation is suggested - as is the practice in France, Spain, and Italy.

Patients in the ICU: all patients should receive prophylaxis. Some countries still give these patients standard prophylaxis (UK, Italy) combined with mechanical prophylaxis (intermittent pneumatic compression), but obese patients or patients with additional risk factors are given intermediate prophylactic doses. France and Spain consider administering therapeutic anticoagulation in patients with very high $\mathrm{D}$-dimer values $(>6 x$ normal value / >3mg/L)

Although the interim ISTH guidelines advise normal prophylactic dosages of heparin (once daily LMWH or twice daily UFH), due to the markedly hypercoagulable present in COVID- I 9 patients, some physicians (see countries above) use intermediate or even full dose (therapeutic) anticoagulation dosages. ${ }^{(22)}$ Anti-Factor $X$ activity (anti-FXa) is not routinely measured for prophylactic anticoagulation therapy and no validated target values are available from clinical trials, but our own experience has indicated that higher doses of LMWH are necessary due to high fibrinogen and high FVIII levels. Antithrombin levels are mostly not very low. Adequate dose titration to achieve antiFXa levels of $0.2-0.5 \mathrm{IU} / \mathrm{ml} 3$ hours after injection may thus be suggested for standard prophylaxis. Intermediate dosing will result in antiFXa levels of $0.4-0.81 \mathrm{U} / \mathrm{mL}$, and to achieve full treatment anticoagulation, the target value of $0.6-1.21 \mathrm{U} / \mathrm{mL}$ is aimed for. Anti-FXa measurement is suggested for all patients when adjusting dosages due to weight, renal dysfunction and increased bleeding risk. Weight based adjustments should be made and effect measured at both ends of the scale, i.e. less than $40 \mathrm{~kg}$ and greater than $100 \mathrm{~kg}$. Renal function must also be monitored in patients receiving LMWH and adjustments must also be made for thrombocytopenia.

In the ICU, especially if inotropic support is required, higher LMWH doses are needed to achieve expected antiFXa levels due to decreased subcutaneous absorption and augmented renal clearance. If UFH are used, better control is achieved by doing anti-FXa levels specific for UFH, than using the activated partial thromboplastin time (aPTT) - especially in the ECMO patient.

Once diagnosed with VTE, initial treatment with LMWH/UFH in hospital with therapeutic anti-FXa levels, and discharge on either LMWH or DOAC.

\section{Type of anticoagulant}

Heparin is known to have anti-inflammatory effects, not only because it decreases thrombin (Flla), but because it binds to inflammatory cytokines, inhibits neutrophil chemotaxis, sequestrates acute phase proteins and neutralizes complement factor 5a. ${ }^{(26)}$ Heparin also has a potential antiviral effect by interacting with the spike proteins on the COVID- 19 virus.(27) For all these reasons heparin is used as an anticoagulant rather than novel oral anticoagulants. Low molecular weight heparin $(\mathrm{LMWH})$ is most often used as there is a much lower risk of heparin-induced thrombocytopenia with thrombosis syndrome (HITTS), unless renal dysfunction is present or there is an expected need for temporary discontinuation related to procedures, where unfractionated heparin may be used. Once discharged or stable the new oral anticoagulants or LMWH may be used, reducing the risk of hospital/clinic visits for INR control.

\section{How long should patients receive anticoagulation?}

Extended prophylaxis for medical patients comes with a risk of bleeding, but in those with elevated VTE risk, up to 45 days may be considered. ${ }^{(25)}$ An increase in D-dimer value $(>2 x$ normal) has been suggested as a marker for thrombotic risk and an indication for extended VTE prophylaxis in medical patients, ${ }^{(28)}$ and may aid in decision-making on when discontinuation of prophylaxis can occur.

Patients that developed VTE during their hospital stay should be treated for the normal period, and D-dimer with patient's history and clinical picture may guide the decision on discontinuing anticoagulation.

Which laboratory tests are recommended in monitoring COVID-I 9 patients?

Depending on resources available, different tests may be performed and due to the rapid emerging information, these suggestions may change:

- Patients at risk for developing severe disease: D-dimer, prothrombin time (PT), FBC [platelet count, lymphocyte and neutrophil counts (neutrophil/lymphocyte ratio)], ferritin, CRP, LDH and IL-6 (IL-6/lymphocyte ratio). ${ }^{(29)}$

Hospitalised patients: as above, as well as activated partial thromboplastin time (aPTT), fibrinogen, U\&E and creatinine, liver enzymes, cardiac markers, PCT, and anti-Factor $X$ activity measurement of LMWH/UFH levels (both in prophylactic and therapeutic dosages).

Patients in ICU: as above. In addition, we find viscoelastography testing on whole blood helpful, particularly thrombo-elastogram (TEG), especially with and without heparinse. Many patients may have prolonged PT values and a hypercoagulable TEG confirms the hypercoagula- 
bility - despite prolonged PT values. A TEG performed with heparinase also provides additional information on other factors involved, such as platelet function (a-angle, maximal amplitude), fibrinogen level (a-angle, maximal amplitude), and presence of fibrinolysis (\% lysis). TEG may be helpful to distinguish DIC from the severe hyperinflammatory coagulation abnormality of COVID-19,(30) and may help in the decision as to whether to use anticoagulation in this "SIC/DIC" state (see above). A decrease in platelet count should always be investigated, as patients do not commonly develop severe thrombocytopenia. Heparin-induced thrombocytopenia with thrombosis syndrome (HITTS), drug reactions, DIC and immune thrombocytopenia, are just some of the considerations. ${ }^{(31)}$

The minimum suggested tests ${ }^{(32)}$ recommend:

a complete or full blood count;

baseline routine coagulation tests (prothrombin time (PT) and activated partial thromboplastin time (aPTT));

- fibrinogen; and

- D-dimer (or other associated tests such as fibrin/fibrinogen degradation products and fibrin monomers as locally available or supported).

A series of biochemistry and other tests are also suggested, including markers for inflammation, electrolyte disturbance, liver dysfunction, and renal and cardiac damage. If anti-FXa levels are not available, a suggested prophylactic dosage calculation may be made taking the D-dimer value, stage of the disease, and patient's weight into consideration ${ }^{(33)}$ (Figure 4).

\section{How do we diagnose VTE in COVID- 19 patients?}

Computed tomography pulmonary angiography (CTPA) is often not possible as patients may be in isolation or very ill and D-dimer values are very high in all patients. A high index of suspicion for thrombosis is necessary in all COVID- 19 patients at all stages of the disease, as the diagnosis may be challenging, especially in the sick ICU patient with pulmonary thrombosis. Worsening right ventricular function may be assessed with echocardiography, and disproportionate hypoxemia, unexplained right ventricular dysfunction or leg swelling or pain should arouse suspicion of VTE (pulmonary thrombosis or embolism). ${ }^{(34)}$

\section{SUMMARY}

\section{SARS-CoV-2 infection leads to a hypercoagulable state:}

- directly due to direct infection of the endothelial cells, and

- indirectly due to the severe inflammatory response and hypoxia that result from the infection. These lead to the activation of the coagulation cascade, platelet activation, NETosis, endothelial dysfunction and decreased fibrinolysis, with SIC. This process may be localised to the lung (pulmonary intravascular coagulopathy), but may also be systemic (especially with stasis, intravenous lines in the ICU) - leading to all 3 VTE risk factors of Virchow's triad present.

- Markers that predict an increased risk of severe disease include clinical risk factors such as older age, male gender, comorbidities such as hypertension and diabetes, cancer, obesity, and laboratory markers such as lymphopenia (increased neutrophil: lymphocyte ratio), increased D-dimer values, mildly prolonged PT, high FDP values, increased LDH levels, increased IL-6 and increased IL-6: lymphocyte ratio. Many of the clinical factors are associated with underlying endothelial dysfunction in COVID-19 negative patients, as well as with VTE risk.

- Pulmonary thrombosis and pulmonary embolism and arterial thrombosis are seen in these patients, especially during admission, but also during pre-admission and postdischarge, and a high index of suspicion is critical.

There is international variability in preventative measures, depending on access to resources, but as more and more histological (post-mortem) examinations are being reported, the severity of the hypercoagulable state of COVID- 19 is being highlighted, ${ }^{(35)}$ with reports of venous as well as arterial thrombotic events, despite "standard" anticoagulation prophylaxis. ${ }^{(36)}$

- Heparin is known to have anti-inflammatory and anti-viral effects, as well as its anticoagulant effect, and also to be protective of the endothelial cell. Studies have shown that prophylactic heparin may decrease mortality in COVID-19, and as such, it is the anticoagulant of choice. Measuring antiFXa activity levels is suggested for both prophylactic and therapeutic management, for both LMWH and UFH. Higher doses of LMWH and aiming for at least intermediate prophylactic anti-FXa levels may prove vital in COVID-19 in patients with additional risk factors or very high D-dimer values. Prophylaxis with LMWH aiming at therapeutic anti-Fa levels should be considered in the severely hypercoagulable patient with $\mathrm{D}$-dimer values of $>3 g / L$ in the absence of bleeding risk. Renal function and platelet count must always be taken into account when prescribing anticoagulation.

\section{Conflict of interest: none declared.}




\section{REFERENCES}

I. WHO timeline-COVID-19. Retrieved 27 April 2020. https://www.who.int/ news-room/detail/27-04-2020-who-timeline---covid- 19.

2. Worldometer. Retrieved 20 May 2020. https://www.worldometers.info/ coronavirus.

3. Fan BE, Chong VCL, Chan SSW, et al. (2020), Haematologic parameters in patients with COVID-19 infection. Am J Hematol, 95:EI 3 I-EI 34.

4. Zhao $X$, Zhang B, Li P, et al. Incidence, clinical characteristics and prognostic factor of patients with COVID-19: A systematic review and meta-analysis. medRxiv 2020.03.17.20037572 (retrieved 22 April 2020, ahead of print, non-peer reviewed).

5. Richards G, Mer M. Schleicher G. Stacey S. COVID-19 and the Rationale for Pharmacotherapy: A South African Perspective. Wits Journal of Clinical Medicine, 2020;2(SI): I I- I8.

6. Lillicrap D. (2020), Disseminated intravascular coagulation in patients with 2019-nCoV pneumonia. J Thromb Haemost, 18:786-787.

7. Tang N, Li D, Wang $X$, Sun Z. Abnormal coagulation parameters are associated with poor prognosis in patients with novel coronavirus pneumonia. J Thromb Haemost. 2020; 1 8:844-847.

8. Cui S, Chen S, Li X, Liu S, Wang F. Prevalence of venous thromboembolism in patients with severe novel coronavirus pneumonia. J Thromb Haemost. 2020; 1 8: | 42 | - | 424

9. Klok FA, Kruip MJHA, van der Meer NJM, et al. Incidence of thrombotic complications in critically ill ICU patients with COVID-19. Thromb Res. 2020; 191:145-147.

10. Oxley TJ, Mocco J, Majidi S, et al. Large-vessel stroke as a presenting feature of COVID-19 in the Young. NEJM 28 April 2020.

II. Fogarty H, Townsend L, Ni Cheallaigh, et al. COVID-19 Coagulopathy in Caucasian patients. (2020), Br J Haematol, 189: I044-1049. Br J Haematol.

2. Tang N, Bai H, Chen X, Gong J, Li D, Sun Z. Anticoagulant treatment is associated with decreased mortality in severe coronavirus disease 2019 patients with coagulopathy. J Thromb Haemost. 2020; I 8: I 094- 1099.

13. Lax SF, Skok K, Zechner P, et al. Pulmonary arterial thrombosis in COVID-19 with fatal outcome: Results from a Prospective, Single-Center, Clin. copathologic Case Series. Ann Intern Med. 2020; 173(5):350-36I. Epub 2020 May 14 .

14. Wichmann D, Sperhake J-P, Lutgehetmann M, et al. Autopsy findings and venous thromboembolism in patients with COVID-19. Annals of Internal Medicine 2020; 173:4,268-277.

15. Najem MY, Couturaud F, Lemarié CA. Cytokine and chemokine regulation of venous thromboembolism. J Thromb Haemost. 2020; 18: 1009- 1019.

16. Schulman S. Coronavirus Disease 2019, Prothrombotic Factors, and Venous Thromboembolism. Semin Thromb Hemost. Online published I I May 2020 Retrieved I2 May 2020.

17. Gupta N, ZhaoY-Y, Evans CE. The stimulation of thrombosis by hypoxia. Thrombosis Research. 2019;181:77-83.

18. Thachil J, Srivastava A. SARS-2 Coronavirus - Associated Hemostatic Lung Abnormality in COVID-19: Is It Pulmonary Thrombosis or Pulmonary Embolism? Semin Thromb Hemost. Published online 12 May 2020. Retrieved 14 May 2020.

19. Varga Z, Flammer AJ, Steiger $P$, et al. Endothelial cell infection and endotheliitis in COVID-19. Lancet 2020;395(I0234):1417-1418.

20. Barnes BJ, Androver JM, Baxter-Stoltzfus A, et al; Targeting potential drivers of COVID-19: Neutrophil extracellular traps. I Exp Med 2020;217(6):e20200652

21. Kwaan HC. Coronavirus Disease 2019: The Role of the Fibrinolytic System from Transmission to Organ Injury and Sequelae. Semin Thromb Hemostasis Published online on 5 May 2020. Retrieved 10 May 2020.

22. Iba $T$, Levy JH, Warkentin TE, et al. Diagnosis and management of sepsisinduced coagulopathy and disseminated intravascular coagulation. J Thromb Haemost. 2019;17:1989-1994.

23. Zhang $Y$, Xiao M, Zhang S, et al. Coagulopathy and Antiphospholipid Antibodies in Patients with COVID-19 April 23, 2020 N Engl J Med 2020

24. Gavriilaki E, Brodsky RA. Severe COVID-19 infection and thrombotic microangiopathy: Success does not come easily. British journal of haematology, 2020: 189(6),e227-e230.
25. Thachil J, Tang N, Gando S, et al. ISTH interim guidance on recognition and management of coagulopathy in COVID-19. J Thromb Haemost, 18:1023-1026.

26. Thachil J. The versatile heparin in COVID-19. J Thromb Haemost. 2020; $00: 1-3$.

27. Mycroft-West C, Su D, Elli S, et al. The 2019 coronavirus (SARS-CoV-2) surface protein (Spike) SI Receptor Binding Domain undergoes conformational change upon heparin binding. bioRxiv 2020.02.29.971093.

28. Cohen AT, Spiro TE, Spyropoulos AC, et al. D-dimer as a predictor of venous thromboembolism in acutely ill, hospitalised patients: A subanalysis of the randomised controlled MAGELLAN trial. J Thromb Haemost 2014; 12:479-87.

29. Chen H, Wang J, Su N, et al. Simplified immune dysregulation index: A novel marker predicts 28-day mortality of intensive care patients with COVID-19. Intensive Care Med 46, I645-1647 (2020)

30. Panigada M, Bottino N, Tagliabue P, et al. Hypercoagulability of COVID-19 patients in Intensive Care Unit. A Report of Thromboelastography Findings and other Parameters of Hemostasis. J Thromb Haemost. 2020; 18:1738-1742

31. Brogaard Larsen J. Pasalic L, Hvas A-M. Platelets in Coronavirus Disease 2019. Semin Thromb Hemost. Published online 30 April 2020. Retrieved 10 May 2020

32. Emmanuel J. Favaloro, Giuseppe Lippi. Recommendations for Minimal Laboratory Testing Panels in Patients with COVID-19: Potential for Prognostic Monitoring. Semin Thromb Hemost 2020;46:379-382.

33. Imperial College Healthcare. Thromboprophylaxis and anticoagulation in COVID- 19 infection. V 0.I 08.04.2020 COVID- 19 Treatment Group. http:// pocketicu.com/wp-content/uploads/2020/03/UK- NHS-Anticoagulation.pdf. Retrieved 28 April 2020

34. Cannegieter SC, Klok FA. COVID-19 associated coagulopathy and thromboembolic disease: Commentary on an interim expert guidance. Res Pract Thromb Haemost. 2020;4:439-445

35. Rotzinger D, Beigelman-Aubry C, Von Garnier C, Qanadli S. Pulmonary embolism in patients with COVID-19: Time to change the paradigm of computed tomography, Thromb Res. 190 (2020) 58-59.

36. Brüggemann R, Gietema $H$, Jallah $B$, et al. Arterial and venous thromboembolic disease in a patient with COVID- 19: A case report. Published online I May 2020, Thromb Res. 2020;191:153-155.

37. Patel JP, Roberts LN, Arya R. Anticoagulating obese patients in the modern era. British Journal of Haematology, 20 I ; | 155: 1 37- 149. 